\title{
Effect of fractionated radiotherapy on the parotid gland: an experimental study in Brazilian minipigs
}

\author{
Roberta Targa Stramandinoli-Zanicotti', Laurindo Moacir Sassi ${ }^{2}$, Juliana Lucena Schussel ${ }^{3}$, Maria Fernanda Torres ${ }^{4}$, \\ Melissa Funchal ${ }^{5}$, Gustavo Henrique Smaniotto ${ }^{6}$, José Luis Dissenha ${ }^{7}$, Andre Lopes Carvalho ${ }^{8}$.
}

1) DDS, MSc, PhD student. Oral and Maxillofacial Surgery Department, Erasto Gaertner Hospital, Curitiba / PR - Brazil; Oncology Department, University of São Paulo, São Paulo/SP - Brazil.

2) DDS, PhD. Chief of the Oral and Maxillofacial Surgery Department, Erasto Gaertner Hospital, Curitiba / PR - Brazil.

3) DDS, PhD. Oral and Maxillofacial Surgery Department, Erasto Gaertner Hospital, Curitiba / PR - Brazil.

4) DVM, PhD. Experimental Surgery Department, Positivo University, Curitiba / PR - Brazil; Veterinary Medicine of Federal University of Parana, Curitiba / PR - Brazil

5) Medical Physics. Radiotherapy Department, Erasto Gaertner Hospital, Curitiba / PR - Brazil

6) MD. Radiotherapy Department, Erasto Gaertner Hospital, Curitiba / PR - Brazil.

7) DDS. Oral and Maxillofacial Surgery Department, Erasto Gaertner Hospital, Curitiba / PR - Brazil.

8) MD, PhD. Head and Neck Surgery and Otorhinolaryngology Department, Hospital de Cancer de Barretos, Barretos, SP, Brazil; Oncology Department, University of São Paulo, São Paulo/SP - Brazil.

Institution: Oral and Maxillofacial Surgery Department, Erasto Gaertner Hospital

Curitiba / PR - Brazil.

Mailing address: Roberta Targa Stramandinoli-Zanicotti - Hospital Erasto Gaertner - Rua Dr. Ovande do Amaral, 201 - Curitiba / PR - Brazil - Zip-code: $81520-060$ E-mail: robertastramandinoli@yahoo.com.br

This research was funded by grants from the Araucaria Foundation (Curitiba, PR, Brazil), the CAPES Foundation (Ministry of Education of Brazil, Brasília, DF, Brazil), and the National Council for Scientific and Technological Development - CNPq (Brasília, DF, Brazil)

Article received on September 23, 2012. Article accepted on December 3, 2012.

\section{SUMMARY}

Introduction: Radiotherapy (RT) of head and neck neoplasms often damages the salivary glands.

Aim: To examine the pattern of morphologic changes resulting from RT of the head and neck region in minipig parotid glands in a clinical and experimental research setting.

Methods: Twelve 18-month-old male Brazilian minipigs weighing 30-40 kg were selected. Eight minipigs were assigned to the experimental group (group 1) and 4 to the control group (group 2). The RT was performed under general anesthesia at Erasto Gaertner Hospital, Curitiba, Brazil, using an á/â ratio of 2.5. The minipigs from group 1 underwent 3 sessions of irradiation with Cobalt 60 of the head and neck, bilaterally, with 3 exposures of 8 Gy each at 7-day intervals for a total dose of 24 Gy. The animals were sacrificed 12 weeks post-RT.

Results: The irradiated parotid glands displayed reductions in the size and number of acini as well as loss of secretory granules. The presence of fibrosis and loss of parenchyma relative to non-irradiated glands were observed, with an average reduction in volume of $54 \%$.

Conclusions: Our results demonstrate that this model for parotid gland damage resulting from an RT regimen appears to be useful for preclinical large animal studies of RT-induced damage and testing novel potential treatment options. Although recent advances in radiation therapy, such as intensity-modulated radiation therapy, have reduced the dose and limited the field of radiation, considerable salivary gland injury still occurs and can greatly impact the patient's quality of life after cancer treatment. Keywords: Head and Neck Neoplasms; Radiotherapy; Salivary Glands; Xerostomia; Swine, Miniature.

\section{INTRODUCTION}

Radiotherapy (RT) is an important part of head and neck cancer treatment, either as the primary modality or as an adjuvant treatment (2). The salivary glands are usually included in the field of radiation. The radiation field for RT of nasopharyngeal and oropharyngeal cancers often includes $100 \%$ of the parotid gland (13). RT causes acute or early and late toxicities, the severity of which depends on the dose that was required for treatment (2). RT also affects tumoradjacent tissue, including the mucosa, vasculature, muscle, bone, and salivary glands, and these tissues are also affected by RT-related side effects $(3,4,10)$. The complications that occur after RT include dental caries, dysgeusia, dysphagia, candidiasis, and osteoradionecrosis $(2,8,10,13)$. Radiationinduced damage to the salivary glands includes parenchymal fibrosis and secretory hypofunction that leads to xerostomia. The effects of RT on the salivary glands become apparent shortly after treatment and are directly related to the structural damage (13).

Although recent advances in radiation therapy, such as intensity-modulated RT (IMRT), have reduced the dose and limited the field of radiation, considerable salivary gland injury still occurs $(5,13)$.

Radiation-induced damage to the salivary gland has 
been studied in many animal models, including the rabbit, monkey, rat, and miniature pig (minipig) (1,6,8,11-16,18). Development of an animal model for radiation-related salivary gland damage using a human RT protocol may assist further investigations on preventing or minimizing such adverse effects and preserve salivary gland function (13). Minipig parotid glands share many anatomic and physiologic characteristics with human glands (20), including the volume and diameter of the main excretory duct. Previous studies have suggested that this animal model is suitable for functional and structural studies of the salivary gland $(9,13)$.

A previous experimental study (6) investigated the histological changes induced by radiation therapy in the submandibular gland of rats. The animals were subjected to doses of 10 and $15 \mathrm{~Gy}$ and sacrificed 16 or 21 days after the RT. Histological examination with hematoxylin and eosin (HE) staining revealed changes in the size, shape, and pigmentation of the nuclei of the acinar cells. Another study evaluated the the early and late effects of radiation on the salivary glands of rats (12) and observed tissue loss and dysfunction of the salivary glands until 1 year postradiotherapy. The radiation-induced injury was delayed in both the parotid and submandibular glands but was more evident in the first.

An experimental study conducted in India, in which pigs were subjected to daily fractionated irradiation totaling 70 Gy and sacrificed 30 days after the completion of RT, showed significant loss of lung parenchyma, with severe acinar atrophy and interstitial fibrosis, enlargement of the nuclei in the remaining acinar cells, and ductal proliferation and dilatation in the irradiated group. No pathological changes were observed in the parotid and submandibular glands of the control group.

Histological analysis of the changes induced by radiotherapy of the salivary glands in experimental studies is important for clinical management and treatment of patients who need RT of the head and neck. The aim of this study was to examine the morphologic changes to minipig parotid glands resulting from fractionated $\mathrm{RT}$ of the head and neck region.

\section{Materials ANd Methods}

\section{Animals}

Eight 18-month-old male Brazilian minipigs (Minipig Br1, Minipig Pesquisa \& Desenvolvimento, São Paulo, Brazil) weighing 30-40 kg were used in this study. The study was reviewed and approved by The Animal Research
Ethics Committee of the Positivo University, Curitiba, PR, Brazil (001/2009). All minipigs were housed together with free access to food and water. Four minipigs were assigned to the experimental group (group 1) and 4 to the control group (group 2).

\section{Irradiation protocol}

The radiotherapy was performed under general anesthesia at Erasto Gaertner Hospital, Curitiba, PR, Brazil. The minipigs from group 1 underwent 3 sessions of irradiation with Cobalt 60 (Theratron 780C - MDS Nordion, Ontario, Canada) of the head and neck, bilaterally, with 3 exposures of $8 \mathrm{~Gy}$ each at 7-day intervals for a total dose of $24 \mathrm{~Gy}$. To ensure that the location of the radiation was correct, a face mask was made from thermoplastic material (Rolyan Aquaplast Splinting Materials, Sammons Preston, Illinois, USA) and used during the RT (Figure 1). Assuming an á/â ratio of 2.5, this dose is biologically equivalent to approximately 56 Gy, or 28 exposures of 2 Gy each (17). The animals in the control group were not irradiated at any time.

\section{Salivary gland histologic evaluation}

The animals from group 1 were sacrificed 12 weeks post-RT. The parotid glands on both sides were dissected from animals from both groups and their macroscopic features analyzed. The specimens were fixed in 10\% formalin, embedded in paraffin, and sectioned at a thickness of $5 \mu \mathrm{m}$ for hematoxylin and eosin (HE) staining. The specimens were examined using a light microscope at 10 to $40 \chi$ magnification by a single pathologist who analyzed all of the specimens. For each section, 5 fields were selected randomly for quantitative histopathologic assessment of the acinar secretory and ductal tissues. The features analyzed were fibrosis, acinar atrophy, parenchymal loss, striated duct dilation, and intercalated duct proliferation and dilation.

\section{Oral tissue evaluation}

The other oral structures that were within the irradiated area, such as the tongue, gingiva, and lipvermillion, were also dissected, fixed in 10\% formalin, embedded in paraffin, sectioned at a thickness of $5 \mu \mathrm{m}$ for HE staining, and analyzed by light microscopy. The aim was to compare non-irradiated and irradiated structures and search for changes induced by irradiation. The same groups of animals were used, and the histological analysis evaluated basophilic degeneration, blood vessels, inflammatory infiltrates, and submucosa structures such as the minor salivary glands and muscle tissue. 


\section{RESULTS}

Irradiated glands

The irradiated and non-irradiated parotid glands were macroscopically different in size and volume (Table 1, Figure 2).

The irradiated parotid glands showed reductions in the size and number of acini, loss of eosinophilic staining in the cytoplasm, and loss of secretory granules. Most cells also exhibited prominent, enlarged, hyperchromatic nuclei. Fibrosis and loss of parenchyma relative to non-irradiated glands could be observed. Significant ductal changes were evident, the most common of which was ductal dilation that was sometimes accompanied by cellular debris and occasional microliths. A mononuclear inflammatory infiltrate with scattered neutrophils was also present (Figure 3).

All irradiated animals remained generally healthy, appeared to eat well, and exhibited no malaise or other clinically significant adverse reaction to RT except for alopecia in the mandibular region.

\section{DISCUSSION}

Radiation is an important tool for both curative and palliative treatment of head and neck cancer. However, despite the beneficial effects of RT on tumor control, the radiation dose to the adjacent normal tissue may cause complications (19). RT for head and neck cancer usually affects the salivary glands, resulting in loss of gland function with reduction in saliva flow and xerostomia as early complications. The loss of saliva flow is a potentially debilitating condition that can permanently compromise oral health and nutrition (13). Radiation-induced xerostomia

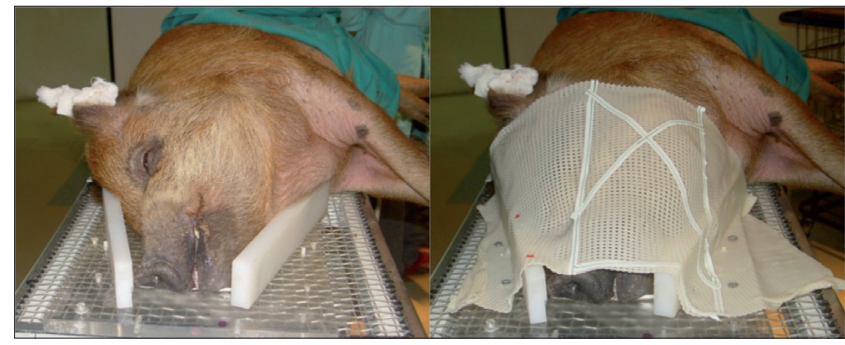

Figure 1. Experimental radiotherapy of the head and neck region in BR-1 Minipigs. A thermoplastic face mask was used for animal immobilization during the radiotherapy sessions.
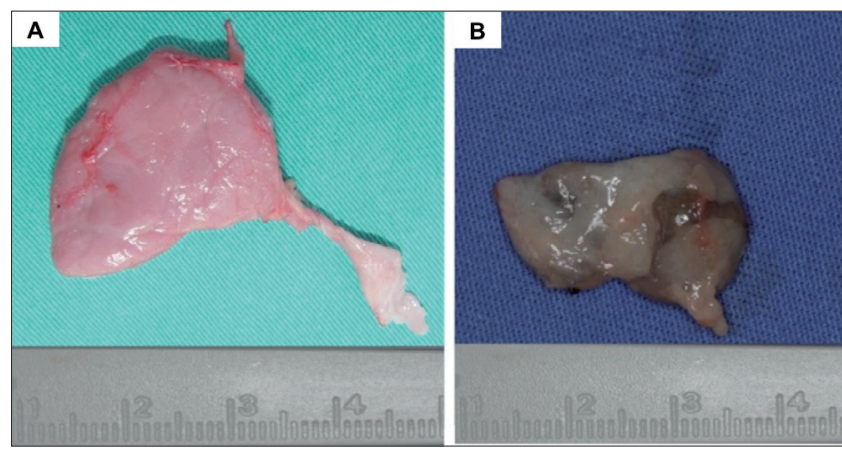

Figure 2. Characterization of the parotid gland after experimental radiotherapy in BR-1 Minipigs: (A) non-irradiated gland and (B) irradiated gland.

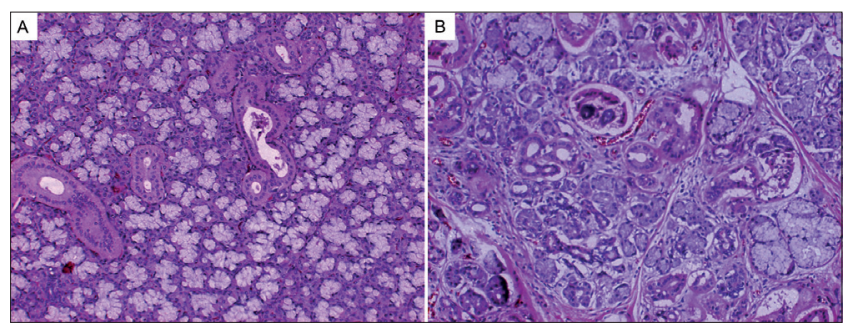

Figure 3. Histological sections of parotid glands from BR-1 Minipigs. (A) Normal parotid gland and (B) Parotid gland after irradiation showing acinar atrophy. (Hematoxylin and eosin, $10 \chi)$.

Table I. Mensuration of irradiated and non-irradiated parotid glands from BR-I Minipigs.

\begin{tabular}{lcccccc}
\hline Sample & & RightGland & Volume & Left Gland & Volume & Average \\
\hline Group I & PI & $5.0 \times 4.2 \times 1.9$ & 39.9 & $4.7 \times 4.4 \times 1.8$ & 37.2 & \\
& P2 & $5.2 \times 4.7 \times 1.1$ & 26.8 & $5.3 \times 3.7 \times 1.4$ & 27.4 & 29.2 \\
& P3 & $5.0 \times 3.9 \times 1.1$ & 21.4 & $4.5 \times 3.2 \times 1.7$ & 24.4 & \\
& P4 & $4.5 \times 3.9 \times 1.6$ & 28.8 & $5.4 \times 4.3 \times 1.2$ & 27.8 & \\
Group2 & P5 & $3.4 \times 2.8 \times 1.5$ & $\mid 4.2$ & $2.8 \times 2.5 \times 1.9$ & 13.3 & \\
& P6 & $3.0 \times 2.9 \times 1.6$ & 13.9 & $3.1 \times 2.4 \times 1.6$ & 11.9 & 13.6 \\
& P7 & $3.0 \times 3.0 \times 1.7$ & $\mid 5.3$ & $3.0 \times 3.0 \times 1.8$ & 16.2 & \\
& P8 & $3.0 \times 2.8 \times 1.5$ & $\mid 3.4$ & $3.5 \times 2.2 \times 1.5$ & 13.9 & \\
& P9 & $3.2 \times 3.0 \times 1.5$ & $\mid 4.4$ & $3.0 \times 2.6 \times 1.5$ & 12.8 & \\
& PI0 & $3.3 \times 2.8 \times 1.5$ & $\mid 3.8$ & $3.2 \times 3.0 \times 1.5$ & 14.4 & \\
& PII & $3.0 \times 3.0 \times 1.5$ & $\mid 3.5$ & $3.0 \times 2.5 \times 1.5$ & 11.2 & \\
& PI2 & $3.2 \times 2.8 \times 1.6$ & $\mid 4.3$ & $3.3 \times 2.5 \times 1.5$ & 12.3 & \\
\hline
\end{tabular}


leads to quantitative and qualitative changes that cause oral dryness. Oral functions are hampered, and the dry and atrophic mucosa can lead to frequent injury (19).

In this study, we used a fractioned RT regimen based on an assumed á/â ratio of 8 Gy with a total dose of 56 Gy delivered over 3 weeks to resemble a human dose protocol. Although it seems likely, we cannot be certain that irradiation would produce a similar effect in humans (17).

Minipigs have been used as a large animal model in medical studies for scientific, economic, and ethical reasons. The oral maxillofacial region of minipigs is similar to that of humans in terms of its anatomy, development, physiology, pathophysiology, and diseases (21). Minipigs are a suitable animal model for functional and structural studies of the salivary glands, and the radiation-induced changes observed in this model are comparable to those observed in humans (13). The minipigs' salivary glands share many characteristics, including their ductal system and gland structure, with those of humans $(20,21)$. Such animal models can help us to understand radiation-related changes better and to facilitate the development of prevention and treatment protocols.

Studies have proven that the alterations in salivary glands functions caused by RT are directly related to the observed morphologic changes $(1,8,13)$. Our results agree with those of previous experimental studies in different animal models, for which there is a consensus that the salivary glands of irradiated animals exhibit macroscopic and microscopic changes. The irradiated salivary glands were reduced in volume by an average of $54 \%$. We were able to observe an important loss of acinar cells as well as gland parenchyma, which is directly related to the decrease in saliva flow and the change in its chemical composition (8). We also confirmed that the salivary glands are the most radiosensitive tissue in the oral cavity. The early and late effects of radiation are related to tissue proliferation. Salivary glands have highly differentiated cells and a slow proliferation rate (7), whereas the tongue, gingiva, and lip vermillion have high rates of cell proliferation and usually experience only early effects of radiation. A similar study demonstrated that a single regional megadose RT protocol induced structural and functional damage to minipig salivary glands. The results of this study showed that the structural changes (i.e., decreased gland weight, acinar atrophy, fibrosis, parenchymal loss, and increased adipose tissue) induced by single and regional megadoses of RT were generally identical to those induced by fractionated radiation dose protocols and similar to those found in humans (8).

The mechanism by which radiation damages the glandular tissue is not entirely known. The theory proposed by Marx (10) suggested that the radiation causes 3 major events in the tissue: hypocellularity, hypovascularity, and hypoxia. Also mentioned are direct cytotoxic effects on acinar cells, as well as side effects consequent to ischemia, obstruction of ducts, and a variety of physiological processes that affect degranulation and secretion-. Hypoxia, which can result from vascular changes, impairs oxidative aerobic cellular respiration, resulting in damage to the cell. Cells can adapt or suffer injury or death depending on the intensity of the hypoxic state. This theory is complemented by the theory of radiation-induced fibrosis (4), which suggests that the activation and dysregulation of fibroblastic activity lead to tissue atrophy within a previously irradiated area. The cells are damaged by acute inflammation, free radicals, chronic activation of fibroblasts, and growth factors.

\section{CONCLUSIONS}

The present study demonstrates that a fractionated RT protocol produces significant RT-induced structural damage in minipig salivary glands. Therefore, this RT regimen model for parotid gland damage appears useful for preclinical large animal studies of RT-induced damage and for testing novel potential treatment options. Although recent advances in radiation therapy, such as intensitymodulated radiation therapy, have reduced the dose and limited the field of radiation, considerable salivary gland injury still occurs and greatly impacts the patient's quality of life after cancer treatment.

\section{ACKNOWLEDGMENTS}

This research was funded by grants from the Araucaria Foundation (Curitiba, PR, Brazil), the CAPES Foundation (Ministry of Education of Brazil, Brasília, DF, Brazil), and the National Council for Scientific and Technological Development-CNPq (Brasília, DF, Brazil).

The authors thank Paola Stramandinoli Branco for her skillful technical assistance. We also express our sincere thanks to the staff members of the Erasto Gaertner Hospital and Department of Experimental Surgery of Positivo University, especially Vanderlei Muller and Cirlei Aparecida Ribeiro do Silva, and to Sheila Albuquerque and Eva Aparecida Rocha for the care of the pigs.

\section{REFERENCES}

1. Ahlner BH, Hagelqvist E, Lind MG, Ruden BI. Irradiation of rabbit submandibular glands. Histology and morphometry after 15 Gy. Acta Otolaryngol. 1993 Mar;113(2):210-9. 
2. Bhide SA, Nutting CM. Advances in radiotherapy for head and neck cancer. Oral Oncol. 2010 Jun;46(6):43941 .

3. Chambers MS, Garden AS, Kies MS, Martin JW. Radiationinduced xerostomia in patients with head and neck cancer: pathogenesis, impact on quality of life, and management. Head Neck. 2004 Sep;26(9):796-807.

4. DelanianS, Lefaix JL. The radiation-induced fibroatrophic process: therapeutic perspective via the antioxidant pathway. Radiother Oncol. 2004;73:119-31.

5. Eisbruch A, Ten Haken RK, Kim HM, Marsh LH, Ship JA. Dose, volume, and function relationships in parotid salivary glands following conformal and intensity-modulated irradiation of head and neck cancer. Int $J$ Radiat Oncol Biol Phys. 1999 Oct 1;45(3):577-87.

6. English JA. Morphologic effects of irradiation on the salivary glands of rats. J Dent Res. 1955;34:4-11.

7. Grundmann O, Mitchell GC, Limesand KH. Sensitivity of salivary glands to radiation: from animal models to therapies. J Dent Res. 2009 Oct;88(10):894-903.

8. LiJ, Shan Z, Ou G, Liu X, Zhang C, Baum BJ, et al. Structural and functional characteristics of irradiation damage to parotid glands in the miniature pig. Int J Radiat Oncol Biol Phys. 2005 Aug 1;62(5):1510-6.

9. Lotz S, Caselitz J, Tschakert H, Rehpenning W, Seifert G. Radioprotection of minipig salivary glands by orciprenalinecarbachol. An ultrastructural and semiquantitative light microscopic study. Virchows Arch A Pathol Anat Histopathol. 1990;417(2):119-28.

10. Marx R. Osteoradionecrosis: a new concept of its pathophysiology. J Oral Maxillofac Surg 1983;41:283-8.

11. Nagler RM, Baum BJ, Miller G, Fox PC. Long-term salivary effects of single-dose head and neck irradiation in the rat. Arch Oral Biol. 1998 Apr;43(4):297-303.

12. Nagler RM. Effects of head and neck radiotherapy on major salivary glands - animal studies and human implications. In Vivo. 2003;17:369-75.
13. Radfar L, Sirois DA. Structural and functional injury in minipig salivary glands following fractionated exposure to 70 Gy of ionizing radiation: an animal model for human radiation-induced salivary gland injury. Oral Surg Oral Med Oral Pathol Oral Radiol Endod. 2003 Sep;96(3):267-74.

14. Sinn DP, Stoker NG, Epker BN. Effects of fractionated doses of cobalt 60 irradiation on rabbit submandibular glands: light microscopic studies. J Oral Surg. 1972;30:277-83.

15. Stephens LC, King GK, Peters LJ, Ang KK, Schultheiss TE, Jardine JH. Unique radiosensitivity of serous cells in rhesus monkey submandibular glands. Am J Pathol. 1986 Sep;124(3):479-87.

16. Veninga T, Visser AG, van den Berg AP, van Hooije CM, van Geel CA, Levendag PC. Equivalence of hyperfractionated and continuous brachytherapy in a rat tumor model and remarkable effectiveness when preceded by external irradiation. Int J Radiat Oncol Biol Phys 2001 Apr 1;49(5):1351-60.

17. Verdonck HW, Meijer GJ, Nieman FH, Stoll C, Riediger D, de Baat C. Quantitative computed tomography bone mineral density measurements in irradiated and nonirradiated minipig alveolar bone: an experimental study. Clin Oral Implants Res. 2008 May;19(5):465-8.

18. Vier-Pelisser FV, Amenábar JM, Cherubini K, Figueiredo MAZ, Yurgel LS. Microscopic analysis of the effect of fractionated radiation therapy on submandibular gland of rats. Radiol Bras 2005;38(6):409-14.

19. Vissink A, Mitchell JB, Baum BJ, Limesand KH, Jensen SB, Fox PC, et al. Clinical management of salivary gland hypofunction and xerostomia in head-and-neck cancer patients: successes and barriers. Int J Radiat Oncol Biol Phys. 2010 Nov 15;78(4):983-91.

20. Wang SL, Li J, Zhu XZ, Sun K, Liu XY, Zhang YG. Sialographic characterization of the normal parotid gland of the miniature pig. Dentomaxillofac Radiol 1998 May;27(3):178-81.

21. Wang S, Liu Y, Fang D, Shi S. The miniature pig: a useful large animal model for dental and orofacial research. Oral Dis. 2007 Nov;13(6):530-7. 